\title{
Les sous-cultures disciplinaires en formation initiale à l'enseignement secondaire au Québec. Un objet de recherche et de formation à problématiser?
}

\section{Frédéric Saussez}

"To some extent each subject in the secondary school is a separate microcosm, a micro world with varying values and traditions" (Goodson \& Mangan, 1995, p. 615)

Cette recherche problématise la notion de sous cultures disciplinaires à partir de différentes recherches anglo américaines conduites dans les champs de l'analyse de l'implantation de politiques éducatives et de l'apprentissage de l'enseignement. Sur cette base, elle questionne la socialisation des futurs enseignants de l'école secondaire québécoise à des sous cultures propres aux disciplines à enseigner. Elle a pour but de mettre à l'épreuve l'hypothèse selon laquelle les conceptions de futurs enseignants de l'école secondaire québécoise à propos du savoir à enseigner varient selon l'appartenance disciplinaire de ceux-ci. Une enquête par questionnaire a été conduite auprès de futurs enseignants de mathématiques et de français. Une analyse factorielle (ACP) a été menée auprès de 109 étudiants inscrits dans un programme de formation menant à l'enseignement des mathématiques $(N=33)$ et de français $(N=76)$. Trois facteurs ont été dégagés. Une ANOVA conduite sur les scores factoriels indique une différence significative entre les futurs enseignants de mathématiques et de français pour chacun de trois facteurs. Sur base de ces résultats, des implications pour la formation à l'enseignement secondaire sont discutées.

Dans le monde francophone de la recherche en éducation, la discipline scolaire est analysée principalement à travers les lunettes de la didactique et de la sociologie du curriculum. Toutefois, elle est encore peu problématisée dans les recherches relatives à l'analyse du travail enseignant à l'école secondaire (Saussez, Lison $\&$ Bouhon, à paraître) et à la formation à l'enseignement secondaire (Saussez, 2009). La visée de cette contribution est d'esquisser quelques pistes de recherche en mesure de donner forme à cette question dans le champ de la formation à l'enseignement secondaire.

Cette contribution s'appuie sur différentes recherches conduites dans le champ de l'analyse de l'implantation des politiques éducatives dans le monde anglo- 
saxon ainsi que dans le champ de l'apprentissage de l'enseignement (Grossman \& Stodolsky, 1994; Mc Laughlin \& Talbert, 2001; Saussez, 2009; Siskin, 1994; Spillane, 2005; Stodolsky \& Grossman, 1995). Celles-ci ont mis en évidence certains des particularismes disciplinaires du travail enseignant à l'école secondaire. Elles indiquent que la discipline à enseigner constitue une composante structurante de l'expérience laborieuse des enseignants de l'école secondaire. Ces recherches soulignent également que la discipline à enseigner constitue souvent un cadre structurant la formation universitaire des futurs enseignants de l'école secondaire. Dans le monde anglo-américain de la recherche en éducation, ces travaux ont contribué à donner forme au problème de sous-cultures propres aux disciplines scolaires. Nous discuterons de manière très schématique de cette question dans la première section.

Le but de cette contribution est de questionner la socialisation des futurs enseignants de l'école secondaire à de telles sous-cultures. De manière spécifique, elle a pour objectif de discuter à l'aide de résultats de recherche, l'hypothèse selon laquelle les conceptions de futurs enseignants de l'école secondaire québécoise à propos du savoir à enseigner varient selon l'appartenance disciplinaire de ceux-ci. Ce sera l'objet de la deuxième section.

\section{De La question de L'organisation de l'école secondaire en départements au problème des sous-cultures disciplinaires}

Au Québec, il y a une caractéristique dans l'organisation de l'école secondaire qui n'est pas problématisée par les travaux sur l'analyse du travail enseignant (voir par exemple Tardif \& Lessard, 1999), à savoir l'existence de départements structurés autour des matières à enseigner. Pourtant, les départements sont dotés de pouvoirs et jouent un rôle dans la structuration des interactions et de la coopération entre les enseignants au sein de l'école. Cette unité organisationnelle, largement ignorée par la recherche en éducation (Siskin, 1994), est en mesure de jouer un rôle important dans la structuration de l'expérience laborieuse des enseignants, la réaccentuation de formes culturelles propres à des groupes sociaux définis en référence à la discipline à enseigner et le filtrage des nouvelles prescriptions véhiculées par les politiques éducatives (Mc Laughlin \& Talbert, 2001; Saussez, 2011). En effet, différents travaux de recherche concernant l'analyse de l'implantation de politiques éducatives conduits en Angleterre, au Canada et aux USA dans les trente dernières années ont mis en lumière l'importance du contexte méso pour la compréhension du processus d'implantation et d'appropriation de nouvelles politiques éducatives à l'école secondaire. Ces travaux indiquent que le département disciplinaire et les sous-cultures disciplinaires constituent des contextes critiques pour comprendre et expliquer le travail enseignant ainsi que la façon dont sont reçus par les enseignants, les projets 
de transformations de l'activité enseignante promus par des réformes éducatives (Saussez et al., à paraître).

Dans cette section, nous discutons sommairement quelques-uns des enseignements que l'on peut tirer de ces travaux de recherche. En effet, ceux-ci ont contribué à donner forme à l'hypothèse selon laquelle la discipline à enseigner constitue le vecteur du développement de sous-cultures disciplinaires spécifiques. Ces travaux constituent la trame dans laquelle s'inscrivent nos préoccupations actuelles de recherche ainsi que les deux ensembles de travaux à la base de la construction des cadres conceptuel et méthodologique structurant notre enquête par questionnaire.

\section{Les rapports complexes entre départements et sous-cultures disciplinaires}

Initialement, le problème de la discipline à enseigner a plutôt été posé dans ce champ, en termes de structures organisationnelles, celles-ci étant en mesure de réifier des particularismes culturels propres aux disciplines à enseigner. Ball (1981, 1987) a observé comment se manifestaient différentes formes de résistance à la réorganisation du regroupement des élèves (passage de groupes de niveau à des groupes hétérogènes) en première année du secondaire au sein d'une école, selon les différents départements disciplinaires et leurs traditions culturelles. Cet auteur a montré que le département constitue un lieu important pour le développement des interactions entre les enseignants (communication et coopération) ainsi qu'une unité administrative impliquée dans la dynamique micro politique de l'école affectant le quoi et le comment enseigner. Il importe donc de ne pas confondre dynamiques départementales et dynamiques propres aux sous-cultures disciplinaires, mais plutôt de tenter de comprendre leurs rapports (Ball \& Lacey, 1995).

Ceux-ci sont complexes. Par exemple, deux départements d'histoire dans deux établissements différents peuvent différer radicalement sur le plan culturel et pourtant, ils peuvent, malgré tout avoir plus en commun qu'un département d'histoire et de math au sein d'un même établissement (Ball \& Lacey, 1995; Siskin \& Little, 1995). Les sous-cultures disciplinaires sont en mesure de différer d'un département à l'autre, notamment en fonction de la dynamique micropolitique à l'œuvre au sein de l'école dont le département constitue justement un élément organisateur (Ball, 1987). Selon les cas, la dynamique départementale est en mesure de pacifier les conflits entre différentes sous-cultures au nom de l'intérêt du département et de ses membres.

Le département constitue donc une unité d'intégration de niveau supérieur aux différentes sous-cultures disciplinaires (Ball \& Lacey, 1995). Il constitue un contexte médiatisant crucial dans la traduction des savoirs curriculaires, dans les rapports enseignant-élèves et la transformation du curriculum prescrit en curriculum énacté. Enfin, il constitue un vecteur important de la construction de l'identité enseignante, de l'engagement vis-à-vis du métier et de la trajectoire 
dans la carrière enseignante (Ball \& Lacey, 1995; Mc Laughlin \& Talbert, 2001; Siskin, 1994).

Les travaux du Center of Research on Teaching in Contectaux USA et notamment, ceux de Mc Laughlin et Talbert (2001, 2006; voir aussi Milbrey, Talbert \& Mc Laughlin, 1993; Talbert, 1995; Talbert \& Mc Laughlin, 1994) contribuent à préciser la façon dont les sous-cultures disciplinaires façonnent le travail enseignant à l'école secondaire. Trois des contextes exerçant un effet structurant sur la culture technique et les normes professionnelles sous-tendant l'activité des enseignants en classe constituent des points d'ancrage aux sous-cultures disciplinaires: le département, les sous-groupes d'enseignants au sein de l'école et les réseaux professionnels en dehors de l'école (association professionnelles, structure de formation continue, etc.).

Tout comme Ball et Lacey (1995), Mc Laughlin et Talbert (2001) montrent que le département est une unité d'intégration de niveau supérieur. Dans les départements monodisciplinaires (math) ou pluridisciplinaire (sciences) où les dynamiques départementales sont fortes, c'est principalement la culture départementale qui alimente la culture technique et les normes professionnelles partagées par les enseignants du département, sans que celles-ci oblitèrent complètement les sous-cultures disciplinaires et l'identification à la discipline à enseigner. Dans le cas où les dynamiques départementales sont faibles, ce sont les sous-cultures disciplinaires qui prennent le dessus, relayées selon les cas par des sous-groupes d'enseignants (par exemple, les profs de chimie au sein d'un département de sciences) ou des réseaux professionnels (associations professionnelles, réseau de formation continue). Ces sous-groupes et réseaux offrent alors un point d'ancrage dans une communauté à des enseignants pour qui les interactions professionnelles sont limitées. Dans ces espaces-temps, les enseignants s'engagent dans des activités socio-discursives à propos de l'exercice du métier, de l'enseignement et de l'apprentissage de la discipline, de nouvelles méthodes, de nouvelles prescriptions, etc. Les sous-cultures disciplinaires fournissent alors un certain nombre de ressources techniques et symboliques en mesure de structurer le noyau technique et les normes professionnelles. C'est dans une telle perspective que ces auteures considèrent que la discipline à enseigner constitue un contexte déterminant du travail enseignant à l'école secondaire. À travers différents relais sociaux, elle donne forme à différents impératifs, à différentes prescriptions concernant l'enseignement et l'apprentissage de la discipline scolaire.

Dans cette perspective, la notion de sous-culture disciplinaire apparaît comme un outil intellectuel particulièrement intéressant pour tenter de mieux comprendre la complexité du travail enseignant à l'école secondaire. Toutefois, il n'en demeure pas moins vrai qu'il s'agit d'une notion dont les contours sont difficiles à cerner. Tout d'abord, il importe de ne pas verser dans une vision essentialiste et homogène des disciplines à enseigner et de leurs cultures en référence exclusivement à des propriétés épistémologiques en les envisageant comme des communautés épistémologiques indifférenciées partageant un corps de savoirs et 
de convictions méthodologiques pour appréhender le monde physique ou social ou encore les réalités éducatives (Ball \& Lacey, 1995). Ball (1981) a observé en effet que les systèmes de normes structurant les sous-cultures disciplinaires ont peu à voir avec des questions épistémologiques mais touchent plutôt à des questions relatives à l'organisation et la transmission des contenus, aux buts de l'enseignement et à l'adhésion à un paradigme pédagogique. Pour Ball (1987, voir aussi Ball \& Cohen, 1999), celle-ci constituent des complexes de valeurs et de présupposés touchant tout à la fois à la nature des savoirs à enseigner et à la façon dont ils doivent s'enseigner ainsi que s'apprendre.

Ensuite, il s'agit de prendre en compte le fait qu'une même discipline à enseigner peut constituer le vecteur du développement au fil du temps, de différentes sous-cultures disciplinaires. On peut donc plutôt les envisager comme des conglomérats de traditions en circulation dans des sous-groupes en conflits ouverts ou larvés (Goodson, 1992). Sous cet angle, les disciplines à enseigner sont des arènes où différents paradigmes disciplinaires (subject paradigms) et pédagogiques (pedagogy paradigm) sont en compétition (Ball, 1987; Ball \& Lacey, 1995). Un paradigme disciplinaire correspond à un système d'idées quant à la nature du savoir à enseigner, à l'organisation des contenus et aux modes de transmission de ceux-ci. Un paradigme pédagogique correspond quant à lui, à un système d'idées (et de procédures pour juger de leur caractère approprié) concernant des manières de faire la classe et de faire apprendre les élèves. Pour Hargreaves (1994), les paradigmes disciplinaires et pédagogiques constituent des éléments structurant du savoir professionnel des enseignants.

En résumé, les disciplines à enseigner participeraient à un processus de stratification de l'identité et de la culture professionnelle des enseignants de l'école secondaire qu'Hargreaves et Mc Millan (1995) désignent en termes de balkanisation. Elles constitueraient un cadre pour le développement de la coopération entre les enseignants de la discipline, sans exclure pour autant des controverses où sont en jeux des paradigmes divergents; elles contribueraient à l'érection de frontières peu perméables entre des groupes d'enseignants définis en référence à la discipline à enseigner et réifiées sous différentes formes au sein et/ou à l'extérieur de l'établissement. Elles constitueraient un trait définitoire (par exemple, je suis prof de chimie et pas simplement prof à l'école secondaire) et renforceraient par conséquent, l'identité disciplinaire. Elles seraient aussi des instruments de la dynamique micro politique au sein de l'école. Enfin, elles constitueraient tout à la fois les vecteurs du développement de formes culturelle façonnant les manières de penser, de ressentir et d'exécuter le travail enseignant et des arènes où différentes sous-cultures peuvent entrer en compétition. 


\section{Du problème des sous-cultures disciplinaires à l'école secondaire à une recherche sur les conceptions du savoir à enseigner en formation initiale à l'enseignement secondaire}

Les travaux évoqués dans le point précédent proposent une vision très générale de la notion de sous-culture disciplinaire. Celle-ci est difficile à opérationnaliser compte tenu de sa multidimensionnalité: modes d'action valorisés, croyances, attitudes, sentiments, normes et valeurs, etc. au regard de différentes composantes de la discipline à enseigner: nature du savoir à enseigner, enseignement et apprentissage de la discipline, buts de l'enseignement, curriculum, etc. Dans cette recherche, nous nous appuyons sur deux ensembles de travaux qui se sont davantage penchés sur les composantes cognitives des sous-cultures disciplinaires: les travaux de Susan Stodolsky et Pamela Grossman (Grossman \& Stodolsky, 1994, 1995; Stodolsky, 1988, 1993; Stodolsky \& Grossman, 1995, 2000).

Cadre conceptuel de la recherche: deux des composantes cognitives d'une sous-culture disciplinaire

Stodolsky et Grossman (1995) ont défendu l'hypothèse que la discipline à enseigner constitue un contexte conceptuel pour les enseignants dans lequel l'enseignant puise des ressources pour construire un contexte d'action. Ainsi, les disciplines à enseigner constituent un contexte conceptuel dans lequel s'orientent les enseignants pour s'engager dans l'activité d'enseignement ou établir un rapport particulier à leur expérience laborieuse (Saussez, 2009). Dans le cadre de différentes recherches, ces auteures se sont plus particulièrement intéressées aux conceptions des enseignants de l'école secondaire à propos de la discipline à enseigner. Cette composante est travaillée à partir de trois dimensions: le degré de définition des contenus de la discipline et de ses frontières en référence à Bernstein (1971), le degré de stabilité des savoirs à enseigner (le fait qu'ils sont stables ou changeants) et le degré de séquentialité des savoirs à enseigner (le fait que la progression dans la discipline est liée à des rapports de succession logique où des contenus sont pré-requis à l'apprentissage d'autres).

Les résultats de ces différentes recherches étayent l'hypothèse que les conceptions des enseignants à propos de la discipline à enseigner varient en fonction de la discipline enseignée. Ils indiquent en outre que ces conceptions fondent bien un contexte conceptuel spécifique à chacune des disciplines. Par ailleurs, ces auteures observent que les pratiques curriculaires déclarées des enseignants (les conceptions du curriculum) varient selon les conceptions de la discipline à enseigner. Ainsi, certaines pratiques curriculaires sont à mettre en rapport avec la conception de la discipline à enseigner. Par exemple, une discipline telle la mathématique, se caractérisant aux yeux de ses enseignants par un haut degré de séquentialité, c'est-à-dire le fait que les contenus de cette discipline reposent les uns sur les autres selon un rapport où un concept ou une capacité est prérequise 
pour en aborder un autre, engendre la perception d'une plus grande coordination à assurer entre les enseignants de cette discipline, d'une plus grande pression à couvrir la matière ou encore d'une plus grande vigilance à ce que les collègues de la discipline font dans leurs cours respectifs.

De Brabander $(1993,2000)$ s'est intéressé plus particulièrement quant à lui aux conceptions des enseignants de l'école secondaire à propos du savoir à enseigner. Il a observé que les enseignants de l'école secondaire avaient une conviction profondément ancrée selon laquelle le savoir qu'ils transmettent se distingue des savoirs transmis au sein des autres disciplines et que celui-ci fonde les différences entre les disciplines enseignées à l'école secondaire (De Brabander, 1993). Pour De Brabander (2000), cette conviction se construit au fil de l'expérience scolaire des personnes lors de leur scolarité secondaire et de la formation disciplinaire à l'université. Celle-ci leur donne, en fait, la légitimité pour enseigner une discipline particulière. De Brabander (1993) formule l'hypothèse que les conceptions du savoir à enseigner constituent une composante centrale des sous-cultures disciplinaires. Par conséquent, ces conceptions devraient varier en fonction des disciplines enseignées par les enseignants à l'école secondaire.

Pour mettre à l'épreuve cette hypothèse, il a mis au point un questionnaire (De Brabander, 2000). La conception du savoir à enseigner est définie en référence à 3 dimensions: 1) l'objet du savoir (en référence à un aspect de la réalité sur lequel il porte par exemple des phénomènes naturels vs des phénomènes culturels), 2) la nature du savoir à enseigner (en référence aux types de concepts qui sous tendent la connaissance dans un champ et aux moyens de l'établir, par exemple un savoir universel, testable et objectif $v$ un savoir particulier, non testable et subjectif), 3) la portée et la fonction du savoir (la portée du savoir est définie en référence à sa sphère d'application par exemple, un savoir général $v s$ un savoir spécifique et la fonction du savoir est définie en référence à son utilité du point de vue de l'élève).

Dans le cadre d'une enquête par questionnaire, cet auteur a mis en évidence deux facteurs organisant les conceptions du savoir à enseigner d'enseignant de l'école secondaire aux Pays-Bas ainsi que les variations au sein de ces conceptions liées à la discipline enseignée par les enseignants. Le premier facteur est interprété par De Brabander (2000) en termes d'opposition entre des savoirs académiques et des savoirs quotidiens. Ce facteurs s'articule autour de trois sous-dimensions: 1) une opposition entre conception dure (un savoir objectif, facilement testable et peu contestable) et douce (un savoir subjectif, difficilement testable et contestable) du savoir à enseigner en référence à la nature de celui-ci ,2) une opposition concernant la fonction du savoir entre une discipline concernée plutôt des apprentissages cognitifs et dont les savoirs ont une valeur pratique ainsi qu'une pertinence pour les études ultérieures et une discipline valorisant des apprentissages diversifiés et dont les savoirs ont une valeur formative ainsi qu'une pertinence pour la vie personnelle des élèves et 3 ) une opposition concernant le mode d'acquisition des savoirs à enseigner entre l'exigence d'une éducation formelle et l'idée que les savoirs à enseigner peuvent s'acquérir dans des situations d'édu- 
cation informelles ou encore entre le fait que l'acquisition de ce savoir requiert des efforts ou non. La seconde dimension est interprétée en référence à une double opposition entre savoir à enseigner général et savoir à enseigner spécifique et entre savoir portant sur des phénomènes culturels ou naturels.

\section{Méthodologie de la recherche et présentation des résultats}

LLa notion de culture présuppose un processus d'appropriation. Bon nombre d'auteurs (Ball, 1987; Borko \& Putman, 1996; Grossman \& Stodolsky, 1994, 1995; Lacey, 1977; Mc Laughlin \& Talbert, 2001; Saussez, 2009; Siskin, 1994; Stodolsky \& Grossman, 1995) envisagent les sous-cultures disciplinaires comme l'objet et le produit d'un long et intense processus de socialisation durant la formation disciplinaire et la formation à l'enseignement à l'université. Toutefois, cette hypothèse n'a jamais été mise à l'épreuve jusqu'à présent.

\section{a) Objectifs de la recherche}

Bien qu'elle ne permette pas de discuter directement cette hypothèse, la recherche que nous présentons constitue un outil pour problématiser la question de sous-cultures propres aux disciplines à enseigner en formation à l'enseignement. L'objectif de cette recherche était d'une part, d'identifier et de décrire les dimensions autour desquelles se structurent les conceptions de futurs enseignants de mathématiques et de français du savoir à enseigner dans le contexte québécois de formation à l'enseignement et d'autre part, de mettre à l'épreuve l'hypothèse selon laquelle ces conceptions varient en fonction de la discipline d'enseignement à laquelle se destinent les personnes. Il s'agit donc de se donner les moyens de répondre à la question suivante: les conceptions de futurs enseignants de l'école secondaire à propos du savoir à enseigner varient-elles en fonction de la discipline dans laquelle ils étudient à l'université?

\section{b) Méthodologie de l'enquête}

Nous avons mené une enquête par questionnaire auprès de futurs enseignants de mathématiques et de français au Québec. Un questionnaire de 38 items a été construit à partir des questionnaires de Stodolsky et Grossman (1995) concernant les conceptions de la discipline à enseigner et de De Brabander (2000) en ce qui a trait aux conceptions du savoir à enseigner. Ce questionnaire a été administré à 109 étudiants en formation initiale à l'enseignement secondaire: 33 futurs enseignants de mathématiques et 76 futurs enseignants de français. Les données sont en cours d'analyse.

Compte tenu du petit nombre de répondants, nous concentrons notre attention dans cette contribution à l'analyse des conceptions du savoir à enseigner. Celles-ci ont été mesurées à l'aide 28 items $^{1}$ (14 paires d'items contrastés) construits à partir du questionnaire de De Brabander (2000). Contrairement à cet auteur qui avait utilisé des échelles bipolaires dans son enquête, nous avons fait le choix 
de transformer chacune de ces échelles en paire constrastée d'énoncés. En effet, lorsque nous avons conçu le questionnaire, nous avons souhaité harmoniser les échelles. Or, celles de Stodolsky et Grossman (1995) se prêtaient peu à une structuration sous la forme de bipôles.

\section{c) Présentation des résultats}

Nous avons procédé à une analyse en composante principale (ACP) sous SPSS avec rotation varimax. L'indice KMO est moyen (.694) et le test de sphéricité de Bartlett est significatif. Nous avons retenus trois facteurs (voir tableau $\mathrm{n}^{\circ} 1$ ) en référence au test du coude de Cattell. Ces trois dimensions expliquent 39,4 $\%$ de la variance.

\section{Tableau 1: Structure des trois facteurs dégagés par ACP (Varimax)}

\begin{tabular}{|c|c|c|c|}
\hline Variables & Facteur 1 & Facteur 2 & Facteur 3 \\
\hline $\begin{array}{l}\text { Q37) Si l'on compare à d'autres disciplines, le curriculum dans } \\
\text { ma discipline est peu défini: l'enseignant est libre d'organiser les } \\
\text { cours en fonction de ses préférences personnelles. }\end{array}$ & ,701 & & \\
\hline $\begin{array}{l}\text { Q12) Dans ma discipline, le savoir est difficilement testable: pour } \\
\text { l'appréciation des performances des élèves, il y a peu de normes } \\
\text { valides universellement qui sont disponibles. }\end{array}$ & ,697 & & \\
\hline $\begin{array}{l}\text { Q6) Dans ma discipline, le savoir concerne essentiellement des } \\
\text { phénomènes socioculturels. }\end{array}$ & ,694 & & \\
\hline $\begin{array}{l}\text { Q 17) Dans ma discipline, le savoir est déterminé subjectivement: } \\
\text { la signification des faits et des idées repose sur des constructions } \\
\text { subjectives. }\end{array}$ & ,670 & & \\
\hline $\begin{array}{l}\text { Q11) Ma discipline offre des apprentissages diversifiés: elle touche } \\
\text { aussi bien des composantes sociales, émotionnelles que cognitives } \\
\text { du développement. }\end{array}$ &, 582 & & \\
\hline $\begin{array}{l}\text { Q9) Dans ma discipline, le savoir consiste principalement à se } \\
\text { forger une opinion: les choses peuvent toujours être regardées sous } \\
\text { un autre angle. }\end{array}$ &, 524 & & \\
\hline $\begin{array}{l}\text { Q 38) Dans ma discipline, l'apprentissage correspond la plupart } \\
\text { du temps à une activité sociale. }\end{array}$ & ,481 & & \\
\hline $\begin{array}{l}\text { Q4) Dans ma discipline, le savoir enseigné peut facilement être } \\
\text { acquis à travers des expériences informelles réalisées en dehors de } \\
\text { l'école. }\end{array}$ & ,356 & & \\
\hline $\begin{array}{l}\text { Q 35) Dans ma discipline, le savoir est facilement testable: } \\
\text { l'appréciation des performances des élèves repose sur des critères } \\
\text { valides universellement. }\end{array}$ &,- 672 & ,370 & \\
\hline $\begin{array}{l}\text { Q 28) Si l'on compare à d'autres disciplines, le curriculum dans } \\
\text { ma discipline est clairement défini: l'enseignant bénéficie d'une } \\
\text { faible marge de manœuvre pour organiser les cours. }\end{array}$ &,- 663 & & \\
\hline $\begin{array}{l}\text { Q 22) Dans ma discipline, le savoir est clairement établi: il s'agit } \\
\text { de faits et d'idées que personne ne peut contester. }\end{array}$ &,- 627 & & ,456 \\
\hline $\begin{array}{l}\text { Q7) Dans ma discipline, le savoir est déterminé objectivement: la } \\
\text { signification des faits et des idées n'est pas sensible aux opinions } \\
\text { subjectives. }\end{array}$ &,- 581 & & \\
\hline
\end{tabular}




\begin{tabular}{|c|c|c|}
\hline $\begin{array}{l}\text { Q 18) Dans ma discipline, le savoir joue un rôle majeur dans la } \\
\text { capacité des élèves à se débrouiller de manière satisfaisante dans } \\
\text { la vie. }\end{array}$ & 681 & \\
\hline $\begin{array}{l}\text { Q3) Dans ma discipline, le savoir a un large champ d'application: } \\
\text { il est utilisé dans des circonstances très différentes. }\end{array}$ & 657 & \\
\hline $\begin{array}{l}\text { Q2) Dans ma discipline, le savoir est plus particulièrement } \\
\text { pertinent pour le développement personnel des élèves et leur vie } \\
\text { privée. }\end{array}$ & ,579 & \\
\hline $\begin{array}{l}\text { Q 34) Dans ma discipline, le principal bénéfice pour les élèves, } \\
\text { c'est un savoir pertinent pour la vie quotidienne. }\end{array}$ & ,460 & \\
\hline $\begin{array}{l}\text { Q 36) Ma discipline offre aux élèves un savoir de base qui les } \\
\text { rend capables de développer une pensée créatrice de manière } \\
\text { autonome. }\end{array}$ &, 424 & \\
\hline $\begin{array}{l}\text { Q25) Dans ma discipline, les élèves trouvent que les cours sont } \\
\text { ardus. }\end{array}$ & ,339 & \\
\hline $\begin{array}{l}\text { Q5) Dans ma discipline, le savoir joue un rôle mineur dans la } \\
\text { capacité des élèves à se débrouiller de manière satisfaisante dans } \\
\text { la vie. }\end{array}$ &,- 608 & ,312 \\
\hline $\begin{array}{l}\text { Q19) Ma discipline offre aux élèves un savoir prêt à l'emploi et } \\
\text { des habiletés qui peuvent être appliquées telles quelles. }\end{array}$ & & \\
\hline $\begin{array}{l}\text { Q13) Dans ma discipline, le principal bénéfice pour les élèves, } \\
\text { c'est un savoir applicable dans les apprentissages scolaires. }\end{array}$ & &, 720 \\
\hline $\begin{array}{l}\text { Q14) Dans ma discipline, le savoir est plus particulièrement } \\
\text { pertinent pour les études ultérieures et le futur métier des élèves. }\end{array}$ & &, 594 \\
\hline $\begin{array}{l}\text { Q 32) Dans ma discipline, l'apprentissage correspond la plupart } \\
\text { du temps à une activité en solitaire. }\end{array}$ &,- 310 &, 586 \\
\hline $\begin{array}{l}\text { Q 33) Ma discipline est dédiée quasi exclusivement au dévelop- } \\
\text { pement cognitif. }\end{array}$ & &, 572 \\
\hline $\begin{array}{l}\text { Q23) Dans ma discipline, le savoir a un champ d'application } \\
\text { limité: il est utilisé dans des circonstances très spécifiques. }\end{array}$ &,- 508 &, 508 \\
\hline $\begin{array}{l}\text { Q 29) Dans ma discipline, le savoir concerne essentiellement des } \\
\text { phénomènes naturels. }\end{array}$ & & ,469 \\
\hline
\end{tabular}

Le premier facteur explique 19,3\% de la variance. Il est structuré autour d'une conception subjectiviste du savoir à enseigner par contraste avec une conception objectiviste de celui-ci. À un premier niveau d'analyse, cette opposition peut être interprétée à partir de la typologie de Biglan (1973) telle que aménagée par Becher (1989) en termes d'opposition entre une vision molle du savoir à enseigner par contraste avec une vision dure de celui-ci. En effet, on observe une forte saturation positive des variables:

- Q 12 (Dans ma discipline, le savoir est difficilement testable: pour l'appréciation des performances des élèves, il y a peu de normes valides universellement qui sont disponibles),

- Q17 (Dans ma discipline, le savoir est déterminé subjectivement: la signification des faits et des idées repose sur des constructions subjectives), 
- Q9 (Dans ma discipline, le savoir consiste principalement à se forger une opinion: les choses peuvent toujours être regardées sous un autre angle).

On observe par contre, une forte saturation négative des variables constituant le pôle opposé de ces trois items:

- Q 35 (Dans ma discipline, le savoir est facilement testable: l'appréciation des performances des élèves repose sur des critères valides universellement),

- Q7 (Dans ma discipline, le savoir est déterminé objectivement: la signification des faits et des idées n'est pas sensible aux opinions subjectives),

- Q 22 (Dans ma discipline, le savoir est clairement établi: il s'agit de faits et d'idées que personne ne peut contester).

Ce qui est en jeu à ce niveau, c'est la nature du savoir à enseigner. D'un côté, il s'agit d'un savoir mou, c'est-à-dire un savoir subjectif, sujet à débat et difficilement testable et de l'autre, il s'agit d'un savoir dur, c'est-à-dire un savoir objectif, clairement établi et facilement testable.

En outre, cette opposition entre un savoir à enseigner mou et un savoir à enseigner dur se double d'une opposition en ce qui a trait à la perception du caractère défini ou non du curriculum dans la discipline d'enseignement: la variable Q 37 (Si l'on compare à d'autres disciplines, le curriculum dans ma discipline est peu défini: l'enseignant est libre d'organiser les cours en fonction de ses préférences personnelles) sature positivement ce facteur alors que la variable Q 28 (Si l'on compare à d'autres disciplines, le curriculum dans ma discipline est clairement défini: l'enseignant bénéficie d'une faible marge de manouvre pour organiser les cours) le sature négativement.

Enfin, d'autres variables saturent positivement ce facteur. Ainsi, le savoir à enseigner concerne essentiellement des phénomènes socioculturels (Q 6) et peut facilement être acquis à travers des expériences informelles réalisées à l'extérieur de l'école (Q 4). Ce savoir contribue à des apprentissages diversifiés concernant autant des dimensions sociales, émotionnelles que cognitives du développement (Q 11) et les apprentissages dans la discipline correspondent la plupart du temps à une activité sociale (Q 38).

Ce facteur regroupe donc des variables qui décrivent la nature du savoir à enseigner en référence notamment à son processus de validation, son caractère peu défini ou défini en lien avec son inscription dans une structure curriculaire ainsi que les apprentissages valorisés et les modalités d'acquisition de ce savoir.

Le facteur 2 explique $12,8 \%$ de la variance. Il est structuré autour d'une conception de la pertinence du savoir à enseigner au regard des exigences de la vie quotidienne et du développement personnel des élèves. Ce facteur est, de prime abord, plus difficile à analyser. On observe tout d'abord un contraste entre deux paires d'énoncé: Q 18 (Dans ma discipline, le savoir joue un rôle majeur dans la capacité des élèves à se débrouiller de manière satisfaisante dans la vie) et Q 3 (Dans ma discipline, le savoir a un large champ d'application: il est utilisé dans des circonstances très différentes) saturent positivement ce facteur alors que la 
variables Q 5 (Dans ma discipline, le savoir joue un rôle mineur dans la capacité des élèves à se débrouiller de manière satisfaisante dans la vie) et la variable Q 23 (Dans ma discipline, le savoir a un champ d'application limité: il est utilisé dans des circonstances très spécifiques) le saturent négativement.

On observe donc une opposition entre un savoir à enseigner conçu comme utile dans de nombreuses sphères d'application y compris la vie quotidienne et un savoir à enseigner dont le champ d'application est restreint et de faible utilité pour la vie quotidienne. Par ailleurs, trois variables, saturant positivement ce facteur, permettent de préciser le sens de la pertinence du savoir à enseigner. Elles concernent plus spécifiquement la pertinence personnelle du savoir à enseigner. En effet, le savoir à enseigner est conçu comme plus particulièrement pertinent pour le développement personnel des élèves ( $\mathrm{Q}$ 2) ainsi que pour la vie quotidienne (Q 34). C’est un savoir de base qui rend les élèves capables de développer une pensée créatrice de manière autonome (Q 36). Enfin deux variables saturent assez faiblement ce facteur et ne s'inscrivent pas dans une vision de la pertinence du savoir à enseigner. La première, corrélée positivement, touche à la perception de la difficulté des cours au sein de la discipline (Q 25) et la seconde, corrélée négativement, concerne la vision de l'apprentissage comme une activité solitaire (Q 32).

Le savoir à enseigner est donc conçu comme ayant une pertinence pour la vie personnelle et quotidienne des élèves. En adoptant la terminologie de Bernstein (1971), nous pourrions dire qu'il s'agit d'un savoir faiblement cadré dont les frontières avec la vie quotidienne et les autres sphères d'utilisation du savoir sont très poreuses.

Le facteur 3 explique 7,3\% de la variance. Il est assez clairement structuré autour d'une conception scolaire du savoir à enseigner. On observe une saturation positive de différentes variables qui sont expliquées par une telle conception. Il s'agit d'un savoir applicable dans les apprentissages scolaires (Q13) et plus particulièrement pertinent pour les études ultérieures (Q 14). Ce savoir s'acquiert la plupart du temps à travers une activité en solitaire (Q32). C'est un savoir qui contribue quasi exclusivement au développement cognitif des élèves (Q 33). Son champ d'application est limité (Q 23). Ce savoir est clairement établi (Q 22) et concerne essentiellement des phénomènes naturels (Q29).

Ces résultats préliminaires recoupent dans une large mesure les résultats obtenus par De Brabander (2000). Comme nous l'avons déjà mentionné, cet auteur a dégagé deux dimensions organisant les conceptions d'enseignants en exercice à propos du savoir à enseigner. La première était structurée autour d'une opposition entre savoirs quotidiens et savoirs académiques. Sous cette opposition, cet auteur contrastait tout d'abord une conception molle et une conception dure du savoir à enseigner Nos résultats reproduisent le même contraste. Les savoirs à enseigner sont tantôt perçus comme subjectifs, discutables et non testables, tantôt comme objectifs, clairement établis et testables. Ensuite, cette première dimension expliquait, tout comme notre premier facteur, l'opposition entre une perception peu définie ou définie du curriculum. 
Par contre, nos résultats ne reproduisent que partiellement les autres contrastes mis en lumière par cet auteur le long de cette première dimension. En effet, celle-ci regroupait également l'opposition entre une vision de la discipline à enseigner qui favorise des apprentissages diversifiés par opposition à une discipline à enseigner qui est dédiée au développement cognitif et l'opposition entre une vision d'un savoir qui peut s'acquérir dans des situations informelles par contraste avec un savoir qui requiert une éducation systématique. Dans notre recherche, le facteur 1 ne reproduit pas ces contrastes. Il est bien saturé positivement par la vision d'une discipline qui favorise des apprentissages diversifiés et d'un savoir qui peut s'acquérir dans des situations informelles. Toutefois, les autres variables structurant la première dimension dégagée par De Brabander (2000) se retrouvent éparpillées au sein des facteurs 2 et 3 de notre étude. Nous avons peut-être là un effet lié au type d'échelle que nous avons utilisé. En effet, offrir un choix le long de deux items est moins contraignant pour le sujet que de se positionner le long d'une opposition entre deux pôles contrastés ${ }^{2}$.

La seconde dimension dégagée par De Brabander (2000) était structurée autour de trois oppositions: la première entre des savoirs ayant un large champ d'application (Q 3) ou un champ d'application limité (Q 23), la deuxième entre des savoirs jouant un rôle majeur (Q18) ou mineur (Q 5) dans la capacité des élèves à se débrouiller dans la vie et la troisième le fait que le savoir concerne essentiellement des phénomènes socioculturels (Q6) ou des phénomènes naturels (Q 29). Le deuxième facteur dégagé dans notre étude recouvre partiellement celle-ci en ce qui a trait aux deux premières oppositions. Néanmoins, il regroupe d'autres variables qui viennent accentuer l'idée d'une pertinence personnelle pour l'étudiant du savoir à enseigner (Q 2, Q 34 et Q 36). La caractérisation de l'objet du savoir à enseigner est répartie sur le premier facteur en ce qui a trait au pôle d'un savoir qui concerne des savoirs socioculturels (Q 6) et le troisième facteurs en ce qui concerne le pôle d'un savoir qui concerne des phénomènes naturels (Q 29). Une conception molle du savoir à enseigner concernerait donc plus particulièrement des savoirs socioculturels alors qu'une conception scolaire du savoir à enseigner aurait pour objet un savoir qui concerne des phénomènes naturels.

Le troisième facteur dégagé dans notre étude n'est pas organisé autour d'oppositions. Le regroupement des variables est opéré autour d'une conception scolaire du savoir à enseigner, à l'exception de la variable Q 29 qui concerne l'objet du savoir à enseigner. En fait, ce facteur donne forme à un seul pôle de l'opposition entre savoirs académiques et savoirs quotidiens qui structurait la première dimension dégagée par De Brabander (2000). Sous cet angle, on peut envisager à nouveau l'hypothèse selon laquelle les échelles utilisées dans le cadre de notre étude étant moins contraignantes que celles employées par De Brabander (2000) ont favorisé un certain éclatement des dimensions.

Dans un second temps, nous avons mis en œuvre les moyens pour répondre à la question suivante: la discipline à enseigner explique-t-elle des variations 
dans ces conceptions du savoir à enseigner? Nous avons conduit une ANOVA sous SPSS avec pour variables dépendantes les scores factoriels et pour variable indépendante la discipline (mathématiques vs français). L'ANOVA permet de mettre en évidence une différence significative ( $\mathrm{p} \lessdot .002$ ou $\mathrm{p}<.000)$ entre les deux disciplines pour chacun des trois facteurs (voir tableau ${ }^{\circ} 3$ ). Comme on le constate à la lecture du tableau $\mathrm{n}^{\circ} 3$, les futurs enseignants de français ont des scores factoriels plus élevés que les futurs enseignants de mathématiques pour les trois facteurs. Ceux-ci conçoivent davantage que les futurs enseignants de mathématique le savoir à enseigner comme un savoir mou, peu défini au plan curriculaire et favorisant des apprentissage diversifiés ainsi que comme un savoir pertinent pour la vie quotidienne. Par contre, les futurs enseignants de mathématiques conçoivent davantage que les futurs enseignants de français le savoir à enseigner comme principalement utile pour les apprentissages ultérieurs et pertinents pour la vie scolaire, ce savoir ayant pour fonction principale le développement cognitif.

Tableau 2: moyenne des scores factoriels selon les deux disciplines

\begin{tabular}{|l|l|r|r|r|r|}
\hline & & $\mathrm{N}$ & Moyenne & Ecart-type & Erreur standard \\
\hline Facteur 1 & 1 Français & 72 &, 1974452 &, 72678818 &, 08565281 \\
\hline & 2 Mathématiques & 30 &,- 4738685 & 1,36398091 &, 24902770 \\
\hline Facteur 2 & 1 Français & 72 &, 2662208 &, 77529154 &, 09136898 \\
\hline & 2 Mathématiques & 30 &,- 6389299 & 1,18864887 &, 21701660 \\
\hline Facteur 3 & 1 Français & 72 &, 2825871 &, 76408033 &, 09004773 \\
\hline & 2 Mathématiques & 30 &,- 6782091 & 1,17444402 &, 21442316 \\
\hline
\end{tabular}

Tableau 3: Anova (avec pour variable indépendante, la discipline et pour variable dépendante, les scores factoriels)

\begin{tabular}{|l|l|r|r|r|r|r|}
\hline & & $\begin{array}{c}\text { Somme des } \\
\text { carrés }\end{array}$ & ddl & $\begin{array}{c}\text { Moyenne des } \\
\text { carrés }\end{array}$ & F & Signification \\
\hline Facteur 1 & Inter-groupes & 9,543 & 1 & 9,543 & 10,435 &, 002 \\
\hline & Intra-groupes & 91,457 & 100 &, 915 & & \\
\hline & Total & 101,000 & 101 & & & \\
\hline Facteur 2 & Inter-groupes & 17,350 & 1 & 17,350 & 20,741 &, 000 \\
\hline & Intra-groupes & 83,650 & 100 &, 837 & & \\
\hline & Total & 101,000 & 101 & & &, 000 \\
\hline Facteur 3 & Inter-groupes & 19,549 & 1 & 19,549 & 24,000 & \\
\hline & Intra-groupes & 81,451 & 100 &, 815 & & \\
\hline & Total & 101,000 & 101 & & & \\
\hline
\end{tabular}


Ainsi, les futurs enseignants de français et de mathématiques de notre échantillon manifestent des conceptions différentes du savoir à enseigner. À nouveau, les résultats obtenus recoupent pour une part ceux de De Brabander (2000). Cet auteur a mis en évidence un effet de la discipline sur les conceptions du savoir à enseigner. Plus spécifiquement, cet auteur a montré que les enseignants de mathématiques partageaient une conception plus dure et académique (au sens de scolaire) du savoir à enseigner que les enseignants de néerlandais (langue maternelle aux Pays-Bas). Ces derniers partagent par contre une conception du savoir à enseigner plus ouverte à la pertinence personnelle que les enseignants de mathématiques.

Toutefois, ces résultats sont à considérer avec précaution compte tenu de la petite taille de l'échantillon et du nombre de variables pris en considération. Il s'agit d'une analyse exploratoire dont la fonction première est de décrire les facteurs structurant les conceptions du savoir à enseigner de futurs enseignants de français et de mathématiques et d'explorer des variations au sein de ces conceptions en fonction de la discipline d'enseignement à laquelle se destinent les personnes. Dans le cadre de cette contribution, ils ont pour fonction de nous aider à problématiser la question des sous-cultures disciplinaires dans le champ de la formation à l'enseignement. Que pouvons-nous dès lors en tirer?

\section{Pour ne pas conclure: des pistes pour problé- matiser les sous-cultures disciplinaires en termes de formation et de recherche}

Ces résultats ne permettent pas vraiment de valider l'hypothèse d'un processus de socialisation disciplinaire qui serait à l'œuvre lors de la formation universitaire à l'enseignement. En effet, nous ne pouvons pas exclure l'hypothèse que ces conceptions se sont développées préalablement à la formation à l'enseignement. Il nous semblerait à cet égard particulièrement pertinent d'étudier auprès des élèves de la fin du secondaire et du collège leur conception du savoir enseigné dans les différentes disciplines scolaires. Toutefois, ces résultats nous encouragent à approfondir l'étude de ces conceptions: se consolident-elles ou sont-elles remaniées lors de la formation initiale à l'enseignement? Sont-elle bien la composante centrale comme le suggère De Brabander (2000) d'un processus d'affiliation des futurs enseignants de l'école secondaire à une communauté disciplinaire ou encore comme le suggèrent Ball et McDiarmid (1990), les futurs enseignants apprennent-ils à l'université des idées diverses concernant la nature du savoir à enseigner?

Ces résultats invitent aussi à réfléchir sur les cadres interprétatifs en mesure de structurer la façon dont les futurs enseignants interprètent les savoirs et autres préconisations pédagogiques transmis en formation initiale. En effet, les étudiants ont du mal à cerner la pertinence de ces savoirs. Dans quelle mesure 
ceux-ci ne sont-ils pas en décalage avec les éléments structurant les traditions pédagogiques disciplinaires? Dans quelle mesure celles-ci ne sont-elles pas tout simplement ignorées par les formateurs? Ainsi, les sous-cultures disciplinaires constitueraient des lentilles à travers lesquels les futurs enseignants regardent l'enseignement et l'apprentissage de la matière ainsi que les différentes prescriptions qui leur sont transmises en formation.

Sous cet angle, les résultats de cette recherche constituent peut-être des outils pour alimenter une réflexion sur la façon de didactiser les savoirs pédagogiques en formation à l'enseignement en prenant en compte le tissu vivant des paradigmes propres aux disciplines à travers lesquels ils doivent se frayer un chemin. Ils constituent aussi un appel à des recherches et des collaborations avec les didactiques afin de cerner les différents paradigmes disciplinaires et pédagogiques qui ont traversés l'histoire de l'enseignement de la discipline scolaire.

Il nous semble également important de souligner l'intérêt qu'il y aurait de faire de ces sous-cultures disciplinaires des objets de formation, par exemple en engageant les étudiants dans un travail d'enquête concernant les paradigmes qui ont structuré l'histoire de l'enseignement de la discipline à l'école secondaire ou encore dans un travail de reconstruction de leur biographie en tant qu'apprenant afin d'explorer le processus personnel de construction de ces conceptions (Saussez \& Ewen, 2006), en suscitant des débats sur des thèmes d'actualités dans le champ de l'école secondaire ou encore la façon dont ils perçoivent les multiples projets actuels de transformation de l'école secondaire afin d'observer les normes, valeurs et croyances relatives à l'enseignement et l'apprentissage de la discipline, l'usage et le rôle de celle-ci dans la formation des élèves en jeu selon les disciplines ou encore en analysant le travail de renormalisation et les outils intellectuels mobilisés pour ce faire lors de séance de coanalyse de l'activité au cours de la formation pratique.

Cette dernière suggestion nous ramène au plus près de nos préoccupations actuelles de recherche dont l'objet est le processus de construction de sens en lien avec l'activité matérielle concrète dans laquelle les enseignants de l'école secondaire sont engagés et nous conduit à envisager pour terminer quatre hypothèses d'analyse qui sont autant d'invitation à poursuivre la recherche concernant les sous-cultures disciplinaires à l'école secondaire et la façon dont elles sont appropriées par les enseignants. Dans les différents travaux sommairement présentés dans la première section, nous sommes en présence d'une variété de situations discursives où c'est la discipline qui parle pour faire comprendre à autrui le sens de l'activité d'enseignement, justifier un geste, une façon de faire, etc.

Notre première hypothèse d'analyse est que les différentes disciplines à enseigner à l'école secondaire sont les vecteurs du développement de cultures d'action (Barbier, 2009) spécifiques, c'est-à-dire des ensembles de formes culturelles interreliées «dans lesquelles les acteurs conçoivent et construisent leur action (Barbier, 2009, p. 166)» ou de façon plus spécifique, "des manières en actes, communes à un ensemble de sujets donnés, de penser leur action, de les concevoir et de les 
conduire et plus généralement de construire du sens autour d'elles (p. 166). Les sous-cultures disciplinaires participeraient donc à un processus de stratification de la culture de l'enseignement au sens entendu par Barbier (2009).

Une deuxième hypothèse d'analyse intimement liée à la première conçoit que ces formes culturelles partagées ou débattues au sein d'une communauté disciplinaire s'incarnent dans un langage social, un sociolecte particulier (Saussez, 2009). Il y aurait donc une langue de métier dans l'enseignement présentant des particularismes en fonction de la discipline enseignée.

Une troisième hypothèse d'analyse considère que des éléments de ce sociolecte sont susceptibles d'être convoqués à titre d'instruments par les enseignants lorsqu'ils s'engagent dans l'élaboration de ce que nous nommons un jugement professionnel, c'est-à-dire un travail de la pensée verbale et réflexive à travers lequel un enseignant met en rapport différents éléments de pensée afin de soutenir une position, expliciter les raisons d'un geste professionnel ou encore expliquer un événement. De ce point de vue, l'analyse provoquée et accompagnée de son activité entreprise par un enseignant sous la conduite d'une chercheur, un intervenant ou un formateur pourrait constituer une situation discursive où c'est la discipline qui parle pour justifier des gestes professionnels. Nous souhaitons ouvrir de cette façon, un espace de réflexion pour problématiser la façon dont il est possible de rendre compte de l'engagement identitaire des enseignants de l'école secondaire à travers la façon dont ils disent leur faire (Barbier, 2000).

Notre quatrième hypothèse d'analyse envisage l'idée que l'apprentissage du métier d'enseignant au sens d'apprentissage des gestes et techniques propres à ce corps de métier se double de la construction progressive de la capacité à se rapporter volontairement à son expérience laborieuse à l'aide de différents instruments sémiotiques constitutifs d'une culture d'action propre à la discipline. Autrement dit, en paraphrasant Schneuwly (2008) lorsqu'il se livre à l'analyse de la fonction psychologique de l'écrit sous l'angle du rapport à l'écrit, apprendre le métier d'enseignant, c'est aussi apprendre à en parler, à le ressentir, l'évaluer et le discuter.

Ainsi, la question des particularismes disciplinaires du métier d'enseignant à l'école secondaire pose au moins deux défis à la recherche. Tout d'abord, elle exige de se donner les moyens d'identifier les formes culturelles spécifiques en circulation au sein de ces différents cercles sociaux, de les resituer au regard des dynamiques sociales, historiques, institutionnelles et organisationnelles qui les marquent. Ensuite, elle exige d'expliquer et comprendre la manière dont ces formes façonnent la subjectivité des enseignants. Il s'agit d'un vaste chantier!

\section{Notes}

1 Le tableau en annexe 1 présente les 28 items utilisés regroupés par paires d'énoncés contrastés.

2 Nous avons procédé initialement à une analyse multidimensionnelle (MDS) qui avait permis de dégager deux dimensions structurant l'ensemble des items mesurant la 
conception du savoir à enseigner (Saussez, 2011). Dans un prochain texte, nous traiterons l'ensemble de ces résultats, tout en discutant de la question de la fiabilité de nos échelles. En effet, les alpha de Cronbach obtenus pour l'ensemble des items retenus pour l'analyse factorielle ainsi que pour chacun des facteurs sont faibles (4.365). L'analyse des résultats indique qu'une piste probable pour expliquer cette fiabilité médiocre réside dans le fait que les facteurs 1 et 2 regroupent probablement des construits différents.

\section{Bibliographie}

Ball, D. \& Cohen, D. (1999). Developping practice, developping practionners: Toward a practice based theory of professional education. In G. Sykes \& L. Darling Hammon (Ed.), Teaching as the learning profession. Handbook of policy and practice (pp. 3-32). Jossey Bass: San Francisco.

Ball, D. \& McDiarmid, G. (1990). The subject matter preparation of the teacher. In R. Houston (Ed.), Handbook of research on teacher education (pp. 437-449). New York: Mc Millan.

Ball, S. (1981). Beachside comprehensive: A case study of secondary schooling. Cambridge University Press.

Ball, S. (1987). The micro-politics of the school. Towards a theory of school organization. Methuen: London.

Ball, S. \& Lacey, C. (1995). Revisiting subject disciplines as the opportunity for group action: A measured critique of subject subcultures. In L. Siskin \& W. Little (Ed.), The subject in question (95-122). Teacher College Press: New York.

Barbier, J.-M. (2000). Sémantique de l'action et sémantique d'intelligibilité des actions. Le cas de la formation. In B. Maggi (Éd.), Manière de penser, manière d'agir en éducation et en formation (pp. 89-104). Paris: PUF.

Barbier, J.-M. (2009). Cultures d'action et modes partagés d'organisation des constructions de sens. Anthropologie des connaissances, 1, (4), 163-194.

Becher, T. (1989). Academic tribes and territories. Buckingham: Open University Press.

Bernstein, B. (1971). On classification and framing of educational knowledge. In M. Youg (Ed.), Knowledge and control: New directions for the sociology of education (pp. 47-69). London: MacMillan.

Biglan, A. (1973). The characteristics of subject matter in different academic areas. Journal of Applied Psychology, 57, (3), 195-203.

Borko, H. \& Putman, R. (1996). Learning to teach. In D. Berliner \& R. Calfee (Ed.), Handbook of educational psychology (pp. 673-708). New York: Macmillan.

De Brabander, C. (1993). Subject conception of teacher and school culture. In F. Kieviet \& R. Vandenberghe (Éd.), School culture, school improvement and teacher development (pp. 77-107). Leiden: DSWO Press.

De Brabander, C. (2000). Knowledge definition, subject, and educational track level: Perception of secondary school teachers. American Educational Research Journal, 37, 1027-1058.

Goodson, I. (1992). School subject and the curriculum change. Falmer Press: London.

Goodson, I. \& Mangan, J. (1995). Subject cultures and the introduction of classroom computers. British Educational Research Journal, 21, 613-626.

Grossman, P. \& Stodolsky, S. (1994). Considerations of content and the circumstances of secondary school teaching. Review of Research of Education, 20,179-221.

Grossman, P. \& Stodolsky, S. (1995). Content as context: The role of school subject in secondary school teaching. Educational Researcher, 24, (8), 5-11.

Hargreaves, A. (1994). Changing teachers, changing times. Rewood book: London.

Hargreaves, A. \& McMillan, R. (1995). The balkanization of secondary school teaching. In L. Siskin \& W. Little (Ed.), The subject in question. Teacher College Press: New York. 
Lacey, L. (1977). The socialization of teachers. Colin Lacey: London.

Mc Laughlin, M. W. \& Talbert, J. (2001). Professional communities and the work of high school teaching. University of Chicago Press.

Mc Laughlin, M.W. \& Talbert, J. (2006). Building school-based teacher learning communities: Professional strategies to improve student achievement. New York: Teachers College Press.

Milbrey, J., Talbert, J. \& Mc Laughlin, M. (1993). High school teaching in context. Teacher College Press: New York.

Saussez, F. (2009). Entre disciplines scolaires et disciplines universitaires, l'affiliation des enseignants de l'enseignement secondaire supérieur en devenir à des cultures disciplinaires. In R. Étienne, M. Altet, C. Lessard, L. Paquay \& P. Perrenoud (Éd.), Former des enseignants professionnels à l'université. Quelles tensions? Quelles modalités? Quelles conditions? Bruxelles: De Boeck.

Saussez, F. (2011, juin). Problématiser le filtrage disciplinaire de la prescription et l'orchestration disciplinaire $d u$ travail enseignant. Conférence présentée au colloque international ERGAPE-CRIFPE «Travail enseignant et politiques éducatives», IUFM de Marseille.

Saussez, F. \& Ewen, N. (2006). La démarche d'investigation critique, pierre angulaire d'une formation à des compétences professionnelles ? D'un cadre conceptuel à l'expérimentation de projets de formation. In J. Desjardin, H. Hensler, O. Dezutter \& A. Beauchesne (Éd.), Développer des compétences en enseignement. Quelle place pour la réflexion professionnelle? Les cahiers scientifiques de l'ACFAS, 108, 45-67.

Saussez, F., Lison, C. \& Bouhon, M. (à paraître). Les cultures d'action propres aux disciplines à enseigner, un outil intellectuel pour articuler dynamique sociale et dynamique subjective dans l'analyse du travail enseignant à l'école secondaire. In F. Saussez, F. Saujat \& R. Amigues (Éd.), Développer l'articulation entre dynamique sociale et dynamique subjective en analyse du travail enseignant. Enjeux et perspectives.

Siskin, L. (1994). Realm of knowledge: Academic department in secondary school. Routledge: Oxon.

Siskin, L. \& Little, J. (1995). The subject in question: Departemental organisation and the high school. New York: Teachers College Press.

Spillane, J. (2005). How the subject matters. School leadership and management, 24, (4), 383-397.

Stodolsky, S. (1988). The subject matter. Chicago: University of Chicago Press.

Stodolsky, S. (1993). A framework for subject matter comparisons in high school. Teaching and Teacher Education, 9, 333-346.

Stodolsky, S. \& Grossman, P. (1995). The impact of subject matter on curricular activity: An analysis of five academic subjects. American Educational Research Journal, 32, 227-249.

Stodolsky, S. \& Grossman, P. (2000). Changing student, changing teaching. Teacher College Record, 95, (1), 125-172.

Talbert, J. (1995). Boundaries of teacher professional communities in US high school power and precariousness. In L. Siskin \& W. Little (Éd.), The subject in question (pp. XX-XX). Teacher College Press: New York.

Talbert, J. \& Mc Laughlin, M. (1994). Teacher professionalism in local school context. American Journal of Education, 102, 123-153

Tardif, M. \& Lessard, C. (1999). Le travail enseignant au quotidien. Contribution à l'étude du travail dans les métiers et les professions d'interactions humaines. Les Presses de l'Université Laval.

Mots-clés: Sous cultures disciplinaires, conception du savoir à enseigner, socialisation disciplinaire, formation à l'enseignement secondaire 


\section{Fachliche Subkulturen in der grundständigen Lehrerbildung der Sekundarstufe I in Québec als Forschungsgegenstand?}

\section{Zusammenfassung}

Die Untersuchung thematisiert den Begriff einer fachlichen Subkultur, ausgehend von verschiedenen angloamerikanischen Studien zum Thema der Implementierung von Bildungspolitik. Auf dieser Grundlage hinterfragt sie die Sozialisierung künftiger Lehrpersonen der Sekundarstufe in Québec in eine Art von fachspezifischen Subkulturen. Ziel ist die Prüfung der Hypothese, dass die Lehrkonzepte der künftigen Sekundarlehrpersonen in Québec von ihrer Zugehörigkeit zu einem bestimmten Fach beeinflusst werden. Es wurden eine Fragebogen-Untersuchung bei künftigen Mathematik- und Französischlehrpersonen sowie eine Faktorenanalyse (PCA) bei 109 Studierenden durchgeführt, die entweder das Fach Mathematikdidaktik (N=33) oder Französischdidaktik $(\mathrm{N}=76)$ studierten. Drei Faktoren haben sich aus der Untersuchung herauskristallisiert. Eine Varianzanalyse (ANOVA) zeigt einen signifikanten Unterschied zwischen den künftigen Lehrpersonen der Mathematik und des Französischen für jeden dieser Faktoren. Von diesen Ergebnissen ausgehend, werden die Folgen für die Lehrerausbildung auf der Sekundarstufe zur Diskussion gestellt.

Schlagworte: Fachliche Subkulturen, Lehrkonzepte, fachliche Sozialisation, Lehrerbildung auf Sekundarstufe

\section{Le sotto-culture disciplinari nella formazione iniziale allsinsegnamento secondario in Québec. Un oggetto di ricerca e di formazione da problematizzare?}

\section{Riassunto}

Questa ricerca problematizza la nozione di sotto-cultura disciplinare come appare in diversi studi di area anglo-americana sull'analisi dell'implementazione delle politiche educative e sull'apprendimento della professione docente. Su questp sfondo, la ricerca si concentra sulla socializzazione degli insegnanti neofiti nelle sotto-culture disciplinari. Questo studio si interroga in particolare su quanto gli studenti nella formazione iniziale per l'insegnamento nel secondario abbiano concezioni diverse a proposito della conoscenza da insegnare a seconda della disciplina che studiano all'università. È stato costruito un questionario di 38 scale, che è stato somministrato a 109 studenti: 33 in matematica e 76 in francese. Sono state estratte tre dimensioni di un PCA. Per analizzare l'effetto della disciplina è stato condotto un test ANOVA usando I valori componente come variabile dipendente, ed è stato riscontrato un effetti significativo per le tre dimensioni. L'articolo discute le implicazioni di questi risultati.

Parole chiave: Sotto-culture disciplinari, concezioni del sapere da insegnare, socializzazione disciplinare, formazione all'insegnamento secondario 


\section{Disciplinary sub-cultures in secondary teacher preparation in Québec as an object of research?}

\section{Summary}

This research problematizes the notion of disciplinary sub-cultures drawing on various Anglo-American studies conducted in the field of the analysis of the implementation of educational policy and the analysis of learning to teach. On this basis, this research focuses on beginning teachers' socialization into subjects' subcultures. This study investigates to what extent students in secondary teacher preparation have different conceptions about the knowledge they transmit depending on the discipline they learn at university. A questionnaire of 38 scales was constructed and administered to 109 students: 33 students in mathematics and 76 in French. A PCA suggested the extraction of three dimensions. To analyse the effect of the discipline an ANOVA was conducted using the component scores as dependent variable. A significant effect was found for each of the three dimensions. The pedagogical implications of these findings are discussed.

Keywords: Disciplinary sub-cultures, conception of the knowledge to teach, disciplinary socialization, secondary teacher preparation 


\section{Annexe}

\section{Variables extraites de de Brabander (2000) présentées par paire d'énoncés.}

Q2) Dans ma discipline, le savoir est plus particulièrement pertinent pour le développement personnel des élèves et leur vie privée.

Q3) Dans ma discipline, le savoir a un large champ d'application: il est utilisé dans des circonstances très différentes.

Q4) Dans ma discipline, le savoir enseigné peut facilement être acquis à travers des expériences informelles réalisées en dehors de l'école.

Q5) Dans ma discipline, le savoir joue un rôle mineur dans la capacité des élèves à se débrouiller de manière satisfaisante dans la vie.

Q6) Dans ma discipline, le savoir concerne essentiellement des phénomènes socioculturels.

Q7) Dans ma discipline, le savoir est déterminé objectivement: la signification des faits et des idées n'est pas sensible aux opinions subjectives.

Q9) Dans ma discipline, le savoir consiste principalement à se forger une opinion: les choses peuvent toujours être regardées sous un autre angle.

Q11) Ma discipline offre des apprentissages diversifiés: elle touche aussi bien des composantes sociales, émotionnelles que cognitives du développement.

Q12) Dans ma discipline, le savoir est difficilement testable: pour l'appréciation des performances des élèves, il y a peu de normes valides universellement qui sont disponibles.

Q13) Dans ma discipline, le principal bénéfice pour les élèves, c'est un savoir applicable dans les apprentissages scolaires.

Q19) Ma discipline offre aux élèves un savoir prêt à l'emploi et des habiletés qui peuvent être appliquées telles quelles.

Q25) Dans ma discipline, les élèves trouvent que les cours sont ardus.

Q 28) Si l'on compare à d'autres disciplines, le curriculum dans ma discipline est clairement défini: l'enseignant bénéficie d'une faible marge de manœuvre pour organiser les cours.

Q 32) Dans ma discipline, l'apprentissage correspond la plupart du temps à une activité en solitaire.
Q14) Dans ma discipline, le savoir est plus particulièrement pertinent pour les études ultérieures et le futur métier des élèves.

Q23) Dans ma discipline, le savoir a un champ d'application limité: il est utilisé dans des circonstances très spécifiques.

Q21) Dans ma discipline, le savoir enseigné peut seulement être acquis à travers une éducation systématique et formelle au sein de l'école.

Q 18) Dans ma discipline, le savoir joue un rôle majeur dans la capacité des élèves à se débrouiller de manière satisfaisante dans la vie.

Q 29) Dans ma discipline, le savoir concerne essentiellement des phénomènes naturels.

Q 17) Dans ma discipline, le savoir est déterminé subjectivement: la signification des faits et des idées repose sur des constructions subjectives.

Q 22) Dans ma discipline, le savoir est clairement établi: il s'agit de faits et d'idées que personne ne peut contester.

Q 33) Ma discipline est dédiée quasi exclusivement au développement cognitif.

Q 35) Dans ma discipline, le savoir est facilement testable: l'appréciation des performances des élèves repose sur des critères valides universellement.

Q 34) Dans ma discipline, le principal bénéfice pour les élèves, c'est un savoir pertinent pour la vie quotidienne.

Q 36) Ma discipline offre aux élèves un savoir de base qui les rend capables de développer une pensée créatrice de manière autonome.

Q 30) Dans ma discipline, les élèves trouvent que les cours sont accessibles.

Q 37) Si l'on compare à d'autres disciplines, le curriculum dans ma discipline est peu défini: l'enseignant est libre d'organiser les cours en fonction de ses préférences personnelles.

Q 38) Dans ma discipline, l'apprentissage correspond la plupart du temps à une activité sociale. 